\title{
Adaptive gaze stabilization through cerebellar internal models in a humanoid robot
}

\author{
Vannucci, Lorenzo; Tolu, Silvia; Falotico, Egidio; Dario, Paolo; Lund, Henrik Hautop ; Laschi, Cecilia
}

Published in:

Proceedings of the 6th IEEE RAS/EMBS International Conference on Biomedical Robotics and Biomechatronics

Link to article, DOI:

10.1109/BIOROB.2016.7523593

Publication date:

2016

Document Version

Peer reviewed version

Link back to DTU Orbit

Citation (APA):

Vannucci, L., Tolu, S., Falotico, E., Dario, P., Lund, H. H., \& Laschi, C. (2016). Adaptive gaze stabilization through cerebellar internal models in a humanoid robot. In Proceedings of the 6th IEEE RAS/EMBS International Conference on Biomedical Robotics and Biomechatronics (pp. 25-30). IEEE.

https://doi.org/10.1109/BIOROB.2016.7523593

\section{General rights}

Copyright and moral rights for the publications made accessible in the public portal are retained by the authors and/or other copyright owners and it is a condition of accessing publications that users recognise and abide by the legal requirements associated with these rights.

- Users may download and print one copy of any publication from the public portal for the purpose of private study or research.

- You may not further distribute the material or use it for any profit-making activity or commercial gain

- You may freely distribute the URL identifying the publication in the public portal 


\title{
Adaptive gaze stabilization through cerebellar internal models in a humanoid robot
}

\author{
Lorenzo Vannucci ${ }^{1 *}$, Silvia Tolu ${ }^{2 *}$, Egidio Falotico ${ }^{1 *}$, Paolo Dario $^{1}$, Henrik Hautop Lund ${ }^{2}$, Cecilia Laschi $^{1}$
}

\begin{abstract}
Two main classes of reflexes relying on the vestibular system are involved in the stabilization of the human gaze: the vestibulocollic reflex (VCR), which stabilizes the head in space and the vestibulo-ocular reflex (VOR), which stabilizes the visual axis to minimize retinal image motion. The VOR works in conjunction with the opto-kinetic reflex (OKR), which is a visual feedback mechanism for moving the eye for moving the eye at the same speed as the observed scene. Together they keep the image stationary on the retina.

In this work we present the first complete model of gaze stabilization based on the coordination of VCR and VOR and OKR. The model, inspired on neuroscientific cerebellar theories, is provided with learning and adaptation capabilities based on internal models. Tests on a simulated humanoid platform confirm the effectiveness of our approach.
\end{abstract}

\section{INTRODUCTION}

One of the objectives of the human gaze stabilization mechanism is to keep the image stable on the retina. The vestibular system, combined with other sensory inputs, plays a major role in stabilizing images; this is an essential task for everyday activities. Two main classes of reflexes, which rely on the output of the inertial system, exist: l. the vestibulo-ocular reflex (VOR), which stabilizes the visual axis to minimize retinal image motion; 2 . the vestibulocollic reflex (VCR), which stabilizes the head in space through the activation of the neck musculature in response to vestibular inputs. The VOR compensates for head movements that would perturb vision by turning the eye in the orbit in the opposite direction of the head movements [1]. VOR works in conjunction with the opto-kinetic reflex (OKR), which is a feedback mechanism for moving the eye at the same speed as the observed scene. Together they keep the image stationary on the retina, with VOR compensating for fast movements and OKR for slower ones [2]. Several approaches have been used to model the VOR depending on the aim of the study. In robotics literature we found some controllers inspired with the VOR [3], [4], [5], [6], but only Shibata and Schaal [3] replicated the OKR mechanism and showed the cooperation between ocular movements.

The VCR stabilizes the head based on the inertial input space by generating a command that moves the head in the opposite direction to that of the current headinspace displacement. When the head is rotated in the plane of a semicircular canal, the canal is stimulated and the muscles

1 The BioRobotics Institute, Scuola Superiore Sant'Anna, Viale R, Piaggio 34, 56025 Pontedera, Italy

2 The Center for Playware, Department of Electrical Engineering, Technical University of Denmark, Elektrovej Building 326, $2800 \mathrm{Kgs}$. Lyngby, Copenhagen, Denmark

* These authors contributed equally to this work. are activated. Thanks to this stimulation, a compensatory rotation of the head along the same axis is produced. Each canal activation produces an appropriate reflex response. The VOR involves six extraocular muscles, each pair acts around a single rotation axis. On the other hand, the VCR controls a complex musculature that includes more than 30 muscles controlling pitch, roll and yaw rotations.

In robotics, some head stabilization models already exist. Yamada and colleagues [7] proposed a method for controlling the neck of a snake-like robot in order to stabilize the head against undulations. The controller is based on the rejection of the disturbance of the body on the head using a continuous model. The model proposed by Marcinkiewicz [8], implemented on the AIBO robot, uses a machine learning algorithm able to learn how to compensate for head rotations in the absence of stabilization mechanisms. Gay and colleagues [9] proposed a head stabilization system for a bipedal robot during locomotion controlled by the optical flow information. It is based on Adaptive Frequency Oscillators to learn the frequency and phase shift of the optical flow. Although the system can successfully stabilize the head of the robot during its locomotion, it does not take in consideration the vestibular inputs. The most close to the neuroscientific findings of the VCR are the works proposed by Kryczka and colleagues [10], [11], [12]. They proposed an inverse jacobian controller [10], [11] based on neuroscientific results [13] and an adaptive model based on a feedback error learning (FEL) [12] able to compensate the disturbance represented by the trunk rotations. All the presented models try to reproduce specific aspects of the gaze stabilization behaviour, but none of them can provide a comprehensive model of gaze stabilization, integrating eye stabilization (OKR and VOR) together with head stabilization (VCR).

\section{A. Role of the cerebellum}

Many theories about the function of the cerebellum have been proposed: i.e. it acts as a comparator in a servocontrol loop, it provides a timing mechanism for sensorymotor actions, it sets control parameters, and it represents a learning machine. The latter implicates that the cerebellum is involved in learning voluntary coordinated actions or in forming predictive internal models. A basic learning mechanism could then support many cerebellar functions, including the regulation of the control parameters of the VOR reflex loop. An interesting hypothesis on cerebellar motor learning, based on an experimental study of the rabbit cerebellum, was proposed by Masao Ito [14] who followed the MarrAlbus model [15], [16]. According to Ito, the adjustment of 
the relative strengths of the direct excitatory and indirect inhibitory pathways adaptively modulates the gain of the VOR. Ito asserted that the modulation could be influenced by messages of retinal image slip and the role of the cerebellum is to store the motor memory for the changes in VOR gains. Miles and Lisberger [17] proposed an alternative model. They stated that the role of the cerebellum was not to store memories but rather to compute an instructive signal to guide the plasticity process.

In this work, with the aim of providing an efficient regulation of the gaze system, both models are combined, and a motor memory learning mechanism guided by the error signal is presented.

\section{B. Objective and rationale}

By considering the analysis of neuroscience findings in the previous section, we can conclude that in order to replicate gaze stabilization behaviors found in humans it is necessary and sufficient to be able to replicate the joint effect of VCR for the head and VOR and OKR for the eye. This work presents the first complete model of gaze stabilization based on the coordination of VCR and OKR/VOR and suitable for the implementation on a robotic platform. By inspiration on the aforementioned cerebellar theories, an adaptive learning mechanism is integrated in the model.

\section{GAZE STABILIZATION MODEL}

A bio-inspired feed-forward control architecture is implemented for the VOR-OKR-VCR system. It consists of a feedback or feedforward controller that generates a command to drive the system accordingly to a desired instruction. Then, an internal model is captured through a learning network in order to precisely perform the control of the system by directly referring to it. The input-output relationship of the internal model is adaptively modified by the teaching error signal conveyed by the climbing fibers (CFs). This information is integrated by the Purkinje cell (PC). The $\mathrm{PC}$ receives inputs from many parallel fibres (PFs), which arise from the granule cells (GCs) situated in the granular layer. Besides CFs, the other main afferent of the cerebellar cortex is the mossy fiber (MF) that makes excitatory synaptic contacts mainly with GCs. Porrill and colleagues [18] stated that both accuracy and learning speed could be greatly improved by optimizing the choice of the centres and transforming the internal sensory-motor representation to an optimal basis of receptive fields (RFs). Motivated by that, we addressed the problem embedding a machine learning approach, Locally Weighted Projection Regression (LWPR) [19], that provides an optimal input representation to the cerebellar granular layer (MF-GC). The LWPR spatially exploits localized linear models at a low computational cost through an online incremental learning. Therefore, the prediction process is quite fast allowing real-time learning. The LWPR processing engine and the cerebellum microcircuit system described here have been proposed in [20], [21]. The LWPR incrementally divides the input space into a set of receptive ficlds (RFs) defined by a center $c_{k}$ and a Gaussian area characterized by a positive definite distance matrix $D_{k}$, as shown in Equation 1.

$$
p_{k}=\exp \left(-\frac{1}{2}\left(x_{i}-c_{k}\right)^{T} D_{k}\left(x_{i}-c_{k}\right)\right),
$$

where $x_{i}$ is a data input point, and $k$ is a local model.

For each iteration, the new input, $x_{i}$, is assigned to the closest RF based on its weight activation, and consequently, the center and the kernel width are incrementally updated. The global output $(y)$ of the LWPR is given by the weighted mean of all the predictions $\left(y_{k}\right)$ of the linear local models $(k)$ created (Equation 2). The number of local models increases with the complexity of the input space.

$$
\hat{y}=\frac{\sum_{k=1}^{N} p_{k} \bar{y}_{k}}{\sum_{k=1}^{N} p_{k}}
$$

Each $p_{k}$ signal, driven by the $k$ th PF contribution, originates from the output of MFs which is sent through the GCs that act as filters $\left(G_{k}\right)$. The output ol these filters depends on the whole input sequence, up to the current ( $M$ th) input:

$$
p_{k}=G_{k}\left(x_{1}, x_{2}, \ldots, x_{M}\right), k=1 . . N
$$

The response of the bank of filters $G_{k}$ is used to compute both the cerebellar output $z(t)=u_{c}$, as defined in Equation 4 , and the update of the synaptic weights (5). PC output $z(t)$, is modelled as a weighted linear combination of the $p_{k}(t)$, while the update rule is derived from [18]:

$$
\begin{aligned}
z(t) & =\sum_{k} w_{k} p_{k}(t), \\
\delta w_{k} & =-\beta e(t) p_{k}(t) .
\end{aligned}
$$

where $\beta$ is a small positive learning rate and $e(t)$ is the error signal carried out by the CF. Hereafter, we describe each learning model block in Fig. 1.

\section{A. Head stabilization system}

Inside the VCR internal model, the learning network (LWPR) generates velocity motor commands $\left(u_{v c r t o t}\right)$ for the head plant in order to achieve stabilization. $u_{\text {vcrtot }}$ is the sum between the output of the VCR feedforward controller $\left(e_{v c r}\right)$ and the output of the internal model $\left(u_{v c r}\right)$. The VCR Feedforward Controller is implemented as a PD controller, and its output is computed as a function of the inertial readings $(I n, \dot{I n})$ :

$$
e_{v c r}=k_{p} \cdot I n+k_{d} \cdot \dot{I n} .
$$

The cerebellar weights are updated by the teaching signal $e_{v c r}$. The inputs to the LWPR are the current and desired position and velocity of the robotic head. LWPR incorporates the cerebellar output into the global internal model. In order to provide a proper reference to the VCR internal model, the current value of the external disturbance must be estimated. Using the readings coming from the inertial measurement unit and the current encoder values, the disturbance vector (d) can be estimated using only direct kinematics functions, by subtracting the expected angular rotations given by the 


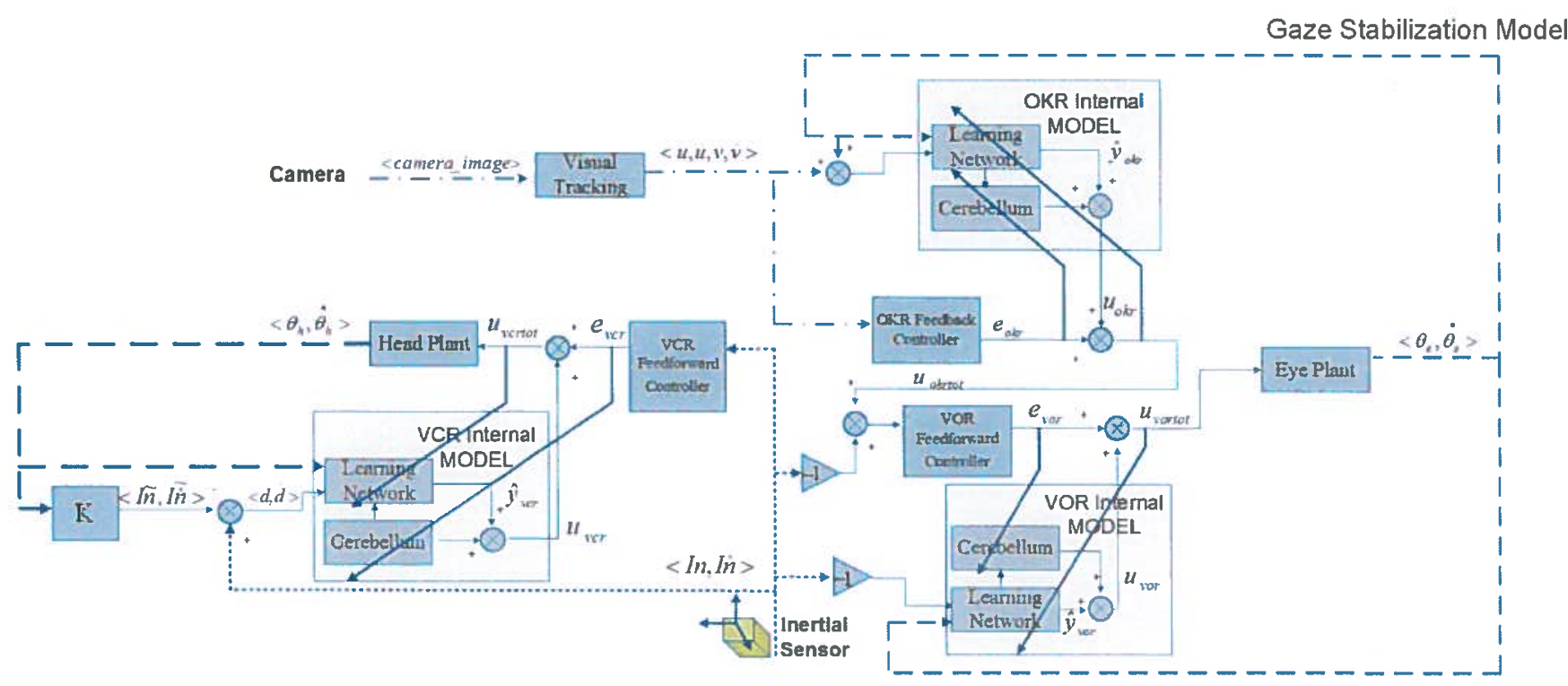

Fig. 1. The proposed model of Gaze stabilization

encoder values (In) from the inertial readings $(I n)$, as follows:

$$
d=I n-\tilde{I n},
$$

where $\tilde{I n}=[\varphi, \vartheta, \psi]$ are the Euler angles for the rigid roto-translation matrix $K\left(\theta_{h}\right)$ from the root reference frame to the inertial frame, computed as:

$$
\begin{aligned}
& \varphi=\operatorname{atan} 2\left(-K\left(\theta_{h}\right)_{2,1}, K\left(\theta_{h}\right)_{2,2}\right), \\
& \vartheta=\operatorname{asin}\left(K\left(\theta_{h}\right)_{2,0}\right) . \\
& \psi=\operatorname{atan} 2\left(-K\left(\theta_{h}\right)_{1,0}, K\left(\theta_{h}\right)_{0,0}\right) .
\end{aligned}
$$

Likewise, the same procedure can be followed in order to estimate the velocity of the disturbance:

$$
\dot{d}=\dot{I n}-\dot{\bar{I} n}=\dot{I n}-J\left(\theta_{h}\right) \cdot \dot{\theta_{h}},
$$

where $J$ is the geometric Jacobian from the root reference frame to the inertial frame.

\section{B. Eye stabilization system}

The eye stabilization system is composed of OKR and VOR subsystems. The position error of the visual target $(u, v)$ and the retinal slip $(\dot{u}, \dot{v})$ enter the OKR stabilization system alongside the eye proprioceptive feedback, with the aim of keeping the image still on the retina. The target position is extracted from the camera image through colour filtering, centroid computation and a conversion from pixels to angles. After this step, the position is derived in order to find the angular velocities in the camera reference frame. On the other hand, the VOR system receives in input the head position and velocity signals, acquired through the vestibular system, and use them as reference to generate appropriate motor commands that compensate for head motion. Moreover, the output of the OKR subsystem is added to the VOR reference signal as a compensatory signal as in [3]. The OKR learning network stores the global internal model given by the sum between the output of the OKR Feedback Controller $\left(e_{o k r}\right)$ and $u_{o k r}$. The cerebellar weights are updated by the teaching signal $e_{o k r}$. The inputs to the learning network are the current and reference position and velocity of the eye, while the error for the PD feedback controller is computed from the target position and velocity $((u, v),(i, \dot{v}))$ :

$$
e_{o k r}=k_{p} \cdot(u, v)+k_{d} \cdot(\dot{u}, \dot{v}) .
$$

Finally, the learning network inside the VOR internal model incorporates the sum between the output of the VOR Feedforward Controller $\left(e_{\text {vor }}\right)$ and the sum of cerebellar and LWPR contributions $\left(u_{\text {vor }}\right)$. The cerebellar weights are updated by the teaching signal $e_{v o r}$, computed as:

$$
\left.e_{\text {vor }}=k_{p} \cdot(-I n)+k_{d} \cdot(-\dot{I n})+u_{\text {okrtot }}\right) .
$$

where $\theta$ and $\dot{\theta}$ are the current eye encoder readings. The inputs to the LWPR are the eye encoders position and velocity $\left(\theta_{e}\right.$ and $\left.\dot{\theta}_{e}\right)$ and the angular position and velocity of the head.

\section{ROBOTIC PlatForm}

The proposed model was implemented for the iCub robot simulator [22], a software included with the iCub libraries. The iCub head contains a total of 6 degrees of freedom: 3 for the neck (pan, tilt and swing) and 3 for the eyes (a common tilt, version and vergence), while the torso has 3 degrees of freedom (pan, tilt and swing). The visual stereo system consists of 2 cameras with a resolution of $320 \times 240$ pixels.

\section{RESUlts}

In order to assess the effectiveness of the proposed model, tests were performed where the robot had to stabilize a target inside the camera image while a disturbance was applied on the head through a torso movement. For this purpose, three kinds of test were performed: 
- compensation of a single axis disturbance, with a moving target;

- compensation of a two axes disturbance, performed with a static target;

- compensation of disturbance which frequency increases over time (linear chirp), after an initial training phase, with a static target.

In all the following experiments the values of the PD gains were set to $k_{p}=4.0, k_{d}=0.1$ for the VCR, $k_{p}=$ $2.0, k_{d}=0.05$ for the VOR and $k_{p}=1.0, k_{d}=0.025$ for the OKR. The Cerebellum module learning rate was set to $\beta=0.005$ for all tests. The distance matrix $D_{k}$ for the LWPR was manually tuned. The motion control loop run at $100 \mathrm{~Hz}$, while camera images were processed at $50 \mathrm{~Hz}$. No information about the internal models was stored between trials, thus the learning phase of the LWPR and Cerebellum modules always started from scratch.

\section{A. Single axis disturbance}

In these tests, sinusoidal disturbances with peak to peak amplitude of $20 \mathrm{deg}$ and frequencies $f \in\{0.5,0.75,1.0$, $1.5,2.0\} \mathrm{Hz}$, were applied on the pitch rotational axis, by moving the robot torso tilt, and a target was placed in front of the robot eyes. In order to see the contribution of the $\mathrm{OKR}$, that stabilize the image on the eye at low frequencies, a sinusoidal motion with a frequency of $0.25 \mathrm{~Hz}$ and a peak to peak amplitude of $10 \mathrm{~cm}$ was applied to the target, on the vertical motion axis.

Results for these tests can be found in Table I, where for each frequency the inertial sensor readings on the pitch axis ( $\operatorname{In}_{p}(\mathrm{deg})$ and $I \dot{n}_{p}(\mathrm{deg} / \mathrm{s})$ ) and the position and speed of the target on the camera image ( $u(\mathrm{deg})$ and $\dot{u}(\mathrm{deg} / \mathrm{s})$ ) are shown. Overlined values indicate the Root Mean Square (RMS), while non-overlined ones indicate the maximum value. In order to avoid having the results corrupted by the initial instability of the learning phase, the maximum and the RMS are computed after a fixed learning period. In particular, only the second half of a 30 s trial was considered. From the results it can be observed that the system is able to compensate for the sinusoidal disturbance with a mean error lower than $4 \mathrm{deg} / \mathrm{s}$ on the camera image for frequencies up to $1.5 \mathrm{~Hz}$. At $2 \mathrm{~Hz}$ frequency the model does not learn fast enough to be able to properly compensate the disturbance after $15 \mathrm{~s}$, but the mean error on the camera image still remains under $5 \mathrm{deg} / \mathrm{s}$.

TABLE I

RESULTS FOR SINGLE AXIS DISTURBANCE.

\begin{tabular}{|c||c|c|c|c|c|c|c|c|}
\hline freq & $I n_{p}$ & $\overline{I n_{p}}$ & $I \dot{n}_{p}$ & $\overline{I \dot{n}_{p}}$ & $v$ & $\bar{v}$ & $\dot{v}$ & $\overline{\dot{v}}$ \\
\hline 0.5 & 0.00 & 0.00 & 0.10 & 0.03 & 1.23 & 0.42 & 3.16 & 0.95 \\
0.75 & 0.01 & 0.01 & 0.32 & 0.09 & 1.06 & 0.50 & 4.22 & 1.20 \\
1.0 & 0.06 & 0.02 & 1.00 & 0.31 & 1.66 & 0.80 & 4.55 & 1.65 \\
1.5 & 0.58 & 0.24 & 7.30 & 2.48 & 1.84 & 0.69 & 6.58 & 2.57 \\
2.0 & 0.90 & 0.44 & 13.55 & 5.96 & 4.45 & 2.61 & 10.99 & 4.08 \\
\hline
\end{tabular}

Figure 2 shows the behaviour of the model during the task at $0.75 \mathrm{~Hz}$ frequency disturbance, in both position and velocity spaces. Values for the disturbance (torso movement), inertial readings and error on the camera image are shown. It can be observed that after an initial learning phase, the error suddenly decreases and the gaze is stable. The model continues to improve the performance over time, thus the error steadily decreases.
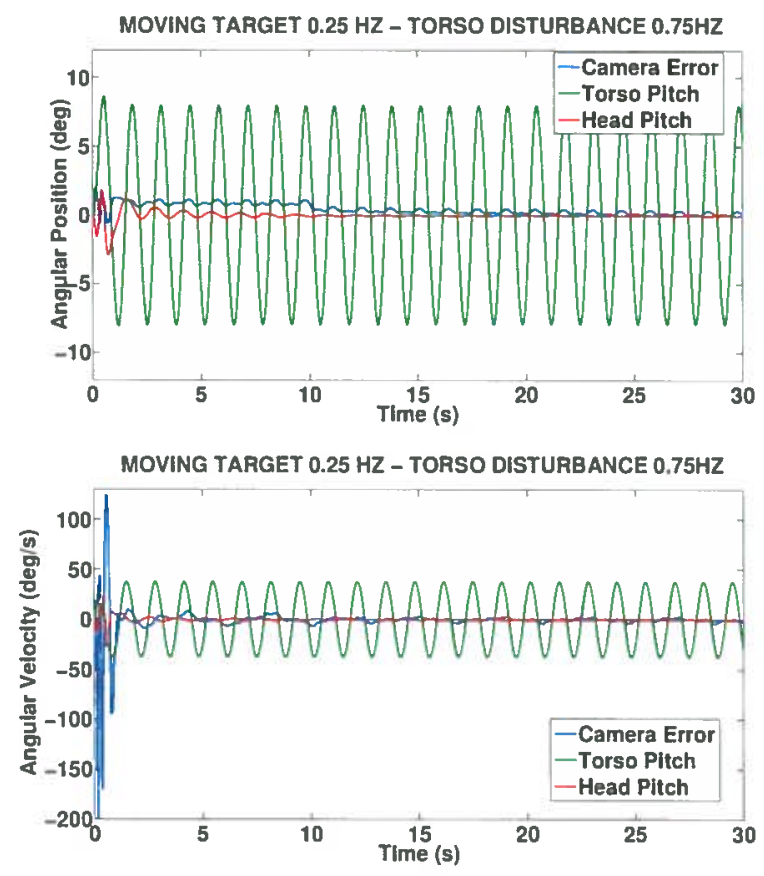

Fig. 2. Results for gaze stabilization during a single axis disturbance position (top) and velocity spaces (bottom).

\section{B. Two axes disturbance}

In these experiments, the same disturbances applied in the single axis case are applied on both the yaw and pitch rotational axes, by moving the torso pan and tilt, while the target remains static during the whole task.

Results for both axes can be found in Table II, where the notation used for Table $I$ is used for the yaw and pitch rotational axes $\left(I n_{y}\right.$ and $\left.I n_{p}\right)$ and camera image axes $(u$ and $v$ ). Compared to the single axis case, the performances decrease due the strong non-linearities of the combination of the disturbances, but the model is still able to properly stabilize the gaze up to $1.0 \mathrm{~Hz}$ disturbance frequency. As in the previous case, only data of the second half of the trial ( $15 \mathrm{~s}$ to $30 \mathrm{~s}$ ) is used to gather measurements for Table II.

Figure 3 shows results for the task at $0.75 \mathrm{~Hz}$ frequency disturbance. As already stated, the error on the vertical axis is larger than in the previous case, due to the influence of the motion on the horizontal axis that adds additional disturbance to the whole system in a non-linear fashion. Nevertheless, the model is able to cope with this disturbance and after the initial learning phase, the gaze is stable and the error continues to decrease during the whole trial.

\section{Linear chirp disturbance}

This test was performed in order to asses the capability of the model to quickly adapt to different motion dynamics. 
TABLE II

RESULTS FOR TWO AXES DISTURBANCE

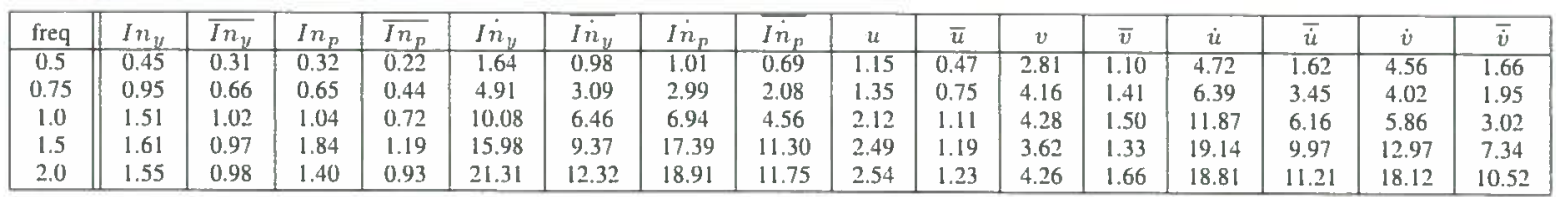
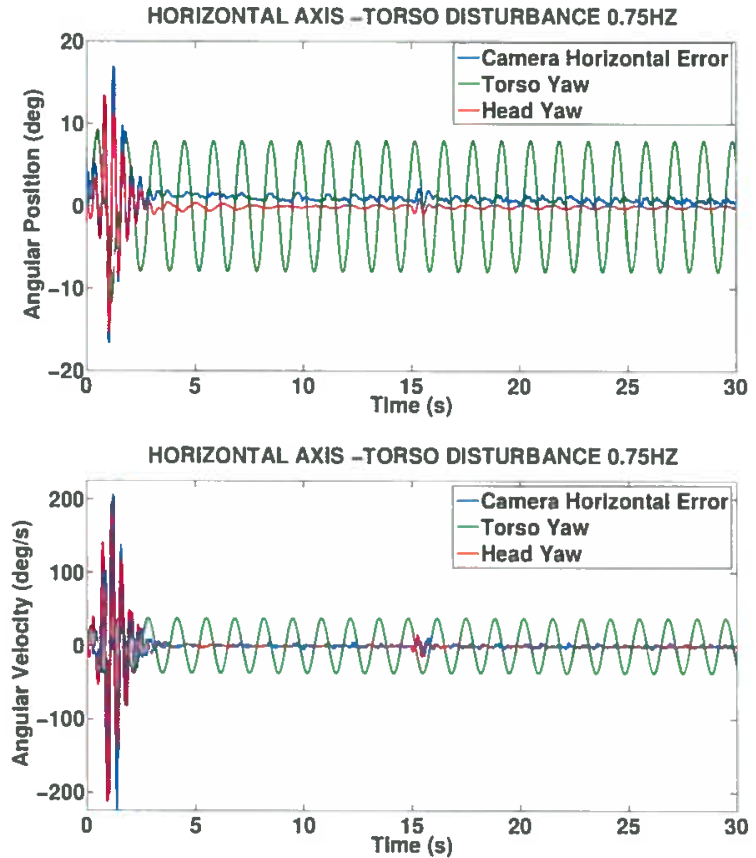
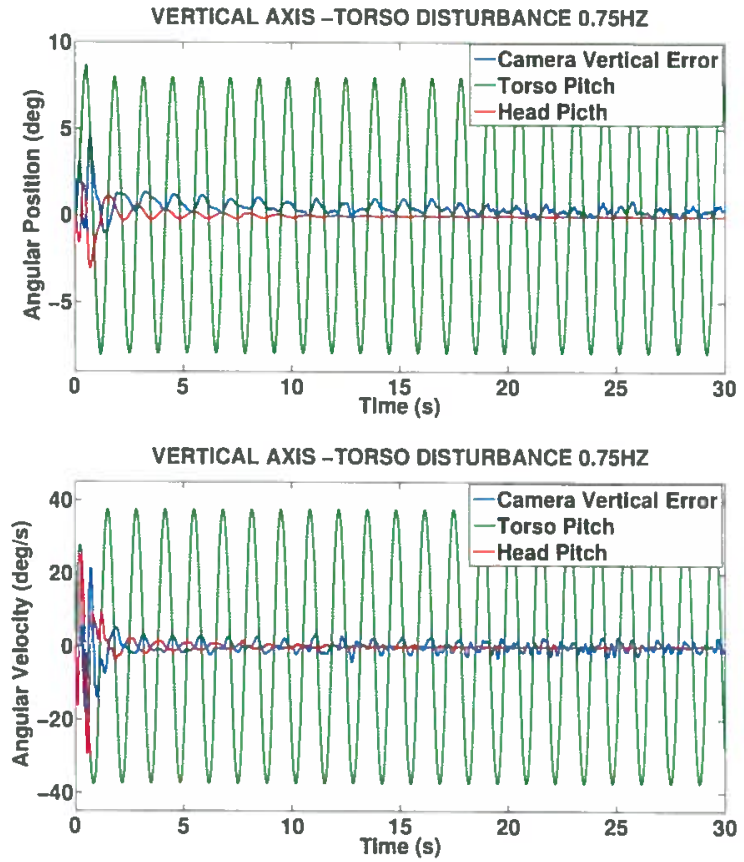

Fig. 3. Results for gaze stabilization during a two axis disturbance. Horizontal (yaw) axis is on the left and vertical (pitch) axis is on the right. Results for position space are in the top row, while results for velocity space are at the bottom.

In the first phase of the trial (14s) a sinusoidal disturbance with $0.5 \mathrm{~Hz}$ frequency was applied on both axes, with a static target. After that phase, the disturbance signal switched to a linear chirp signal with a starting frequency of $0.5 \mathrm{~Hz}$ and a rate of frequency increase of $0.04 \mathrm{~Hz} / \mathrm{s}$.

Results for the trial can be seen in Figure 4. The chirp signal starts after 14 seconds, and reaches a frequency of $1.78 \mathrm{~Hz}$ at the end of the trial. After the initial phase, the error on the camera image remains under a maximum value of $9.05 \mathrm{deg} / \mathrm{s}$ with a RMS of $3.32 \mathrm{deg} / \mathrm{s}$ on the horizontal image axis and under a maximum $3.40 \mathrm{deg} / \mathrm{s}$ and a RMS of $1.22 \mathrm{deg} / \mathrm{s}$ on the vertical image axis.

\section{CONCLUSION}

In this work we present the first complete model of gaze stabilization based on the coordination of VCR and $\mathrm{OKR} / \mathrm{VOR}$ and we validate it through an implementation on a simulated humanoid robotic platform. The presented learning model took advantage of the combination between a machine learning approach and a set of uniform cerebellar circuits. We exploited the LWPR features to incrementally learn and store the inverse internal models of the VCR-VOROKR system, and to map the adapting kerncls for an efficient representation of the input-space with a limited number of them. The C-LWPR module embedded in each block of the gaze system, contributes to deliver finer and niore effective corrections that lead to an optimized internal model learning. The results show that the model is able to reject the torso disturbance on a single axis at different frequencies (up to $2 \mathrm{~Hz}$ ) to stabilize the target on the image even when it is moving. The RMS error after 15 seconds remains under $4 \mathrm{deg} / \mathrm{s}$ except for the $2 \mathrm{~Hz}$ trial. For trials concerning double axis disturbance, the performance deteriorate for frequencies greater than $1 \mathrm{~Hz}$. In future work we will implement a mechanism to store LWPR weights after a learning time in order to reduce the learning time and we will include a stabilization mechanism also for the roll rotational axis. The results obtained in gaze stabilization allow another important future study, concerning walking on soft ground. Walking on soft ground has different effects as shown by [23], [24], [25]. The torso perturbation as well as the head translation (mostly in the vertical plane) change in frequency and amplitude to support step adaptation and stable posture during locomotion. The gaze stabilization controller presented here can compensate for such changes and also ensures a stable head orientation and image stabilization on 

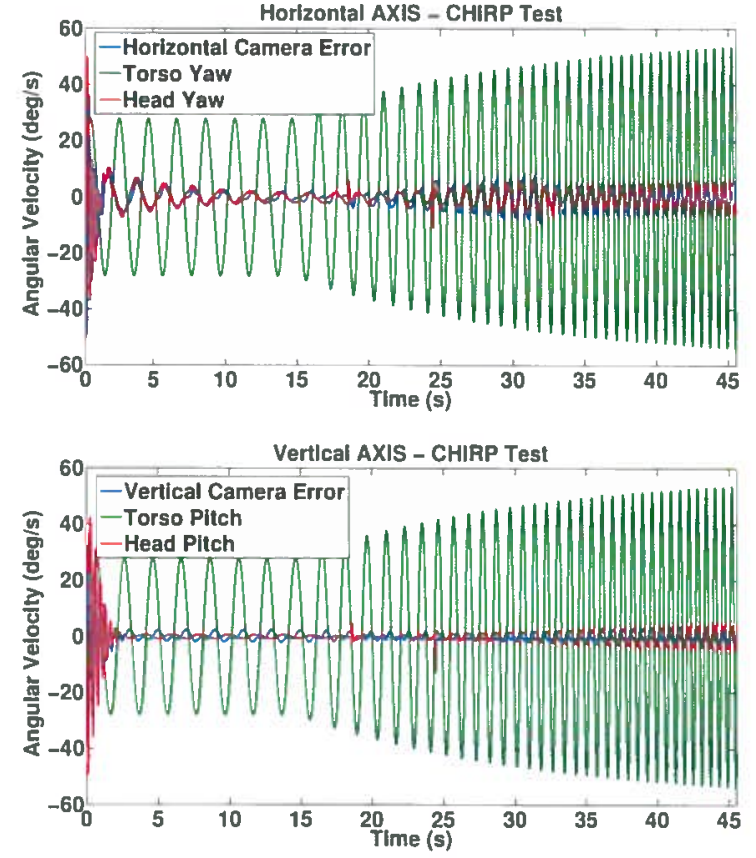

Fig. 4. Results for gaze stabilization during a two axis chirp disturbance on the yaw (top) and pitch rotational axis (bottom), velocity space.

soft ground, too.

\section{ACKNOWLEDGMENT}

The research leading to these results has received funding from the European Union Seventh Framework Programme (FP7/2007-2013) under grant agreement no. 604102 (Human Brain Project). The authors would like to thank the Italian Ministry of Foreign Affairs, General Directorate for the Promotion of the "Country System", Bilateral and Multilateral Scientific and Technological Cooperation Unit, for the support through the Joint Laboratory on Biorobotics Engineering project.

\section{REFERENCES}

[1] G. Barnes, "Visual-vestibular interaction in the control of head and eye movement: the role of visual feedback and predictive mechanisms," Progress in Neturobiology, vol. 41, no. 4, pp. 435-472, 1993

[2] G. Schweigart, T. Mergner, I. Evdokimidis, S. Morand, and W. Becker, "Gaze stabilization by optokinetic reflex (okr) and vestibulo-ocular reflex (vor) during active head rotation in man," Vision Research, vol. 37, no. 12, pp. 1643-1652, 1997.

[3] T. Shibata and S. Schaal, "Biomimetic gaze stabilization based on feedback-error-learning with nonparametric regression networks." Neural Nerworks, vol. 14, no, 2, pp. 201-216, 2001.

[4] S. Viollet and N. Franceschini, "A high speed gaze control system based on the vestibulo-ocular reflex," Robotics and Autonomous Sysiems, vol. 50, no. 4, pp. 147-161, 2005.

[5] J. Porrill, P. Dean, and J. V. Stone. "Recurrent cerebellar architecture solves the motor-error problem." Proceedings of the Roval Society of London-B, vol. 271, no. 1541, pp. 789-796, 2004.

[6] E. Franchi, E. Falotico, D. Zambrano, G. Muscolo, L. Marazzato P. Dario, and C. Laschi, "A comparison between two bio-inspired adaptive models of vestibulo-ocular reflex (VOR) implemented on the iCub robot." in 2010 loth IEEE-RAS International Conference on Humanoid Robots, Humanoids 2010, 2010, pp. 251-256.

[7] H. Yamada, M. Mori, and S. Hirose, "Stabilization of the head of an undulating snake-like robot," in Intelligent Robots and Systems (IROS). 2007 IEEE/RSJ International Conference on, 2007. pp. 3566-3571.
[8] M. Marcinkiewicz, R. Kaushik, I. Labutov, S. Parsons, and T. Raphan, "Learning to stabilize the head of a quadrupedal robot with an artificial vestibular system." in Robotics and Automation (ICRA), 2009 IEEE International Conference on, 2009, pp. 2512-2517.

[9] S. Gay, J. Santos-Victor, and A. Ijspeert, "Learning robot gait stability using neural networks as sensory feedback function for central pattern generators," in Intelligent Robots and Systems (IROS), 2013 IEEE/RSJ International Conference on, Nov 2013, pp. 194-201.

[10] P. Kryczka, E. Falotico, K. Hashimoto, H. Lim. A. Takanishi, C. Laschi, P. Dario, and A. Berthoz, "Implementation of a human model for head stabilization on a humanoid platform," in Proceedings of the IEEE RAS and EMBS International Conference on Biomedical Robotics and Biontechatronics, 2012, pp. 675-680.

[11] P. Kryczka, E. Falotico, K. Hashimoto, H.-O. Lim, A. Takanishi, C. Laschi, P. Dario, and A. Berthoz, "A robotic implementation of a bio-inspired head motion stabilization model on a humanoid platform," in IEEE International Conference on Intelligent Robots and Systems. 2012, pp. 2076-2081.

[12] E. Falotico, N. Cauli, K. Hashimoto, P. Kryczka, A. Takanishi, P. Dario, A. Berthoz, and C. Laschi. "Head stabilization based on a feedback error learning in a humanoid robot," in Proceedings - IEEE International Workshop on Robot and Human Interactive Communication, 2012, pp. 449-454.

[13] E. Falotico, C. Laschi, P. Dario, D. Bernardin, and A. Berthoz, "Using trunk compensation to model head stabilization during locomotion." in IEEE-RAS International Conference on Humanoid Robots, 2011 pp. $440-445$.

[14] M. Ito, "Mechanisms of motor learning in the cerebelluml," Brain Research, vol. 886, no. 12, pp. $237-245,2000$.

[15] D. Marr and W. T. Thach, From the Retina to the Neocorter: Selected Papers of David Marr. Boston, MA: Birkhäuser Boston, 1991, ch. A Theory of Cerebellar Cortex, pp. II-50.

[16] J. S. Albus, "A theory of cerebellar function," Mathematical Biosciences, vol. 10, no. 1-2, pp. 25-61. 1971

[17] F. A. Miles and S. G. Lisberger, "Plasticity in the vestibulo-ocular reflex: a new hypolhesis." Annual review of neuroscience, vol. 4, no. 1, pp. 273-299, 1981

[18] J. Porrill and P. Dean, "Recurrent cerebellar loops simplify adaptive control of redundant and nonlinear motor systems." Neural Computafion. vol. 19. no. I, pp. 170-193, 2007

[19] S. Vijayakumar and S. Schaal, "Locally weighted projection regression: Incremental real time learning in high dimensional space," in ICML '00: Proceedings of the Seventeenth International Conference on Machine Learning. San Francisco. CA. USA: Morgan Kaufmann Publishers Inc., 2000, pp. 1079-1086.

[20] S. Tolu, M. Vanegas, N. R. Luque, J. A. Garrido, and E. Ros, "Bio-inspired adaptive feedback error learning architecture for motor control," Biological Cvbernetics, vol. 106, no. 8-9, pp. 507-522. 2012. Online]. Available: http://dx.doi.org/10.1007/s00422-012-0515-5

[21] S. Tolu, M. Vanegas, J. A. Garrido, N. R. Luque, and E. Ros, "Adaptive and predictive control of a simulated robot arm," Int. J. Neural Syst. vol. 23, no. 3, 2013.

[22] V. Tikhanoff, A. Cangelosi, P. Fitzpatrick, G. Metta, L. Natale, and F. Nori, "An open-source simulator for cognitive robotics research: The prototype of the icub humanoid robot simulator," in Proceedings of the 8th Workshop on Performance Metrics for Intelligent Svstems. ser. PerMIS '08. New York, NY, USA: ACM, 2008. pp. 57-61. Online]. Available: http://doi.acm.org/10.1145/1774674.1774684

[23] M. J. MacLellan and A. E. Patla, "Adaptations of walking pattern on a compliant surface to regulate dynamic stability." Experimentual Brain Research, vol. 173, no. 3, pp. 52I-530, 2006.

[24] H. i. Kang, K. Hashimoto, K. Nishikawa, E. Falotico, H. o. Lim. A. Takanishi, C. Laschi, P. Dario, and A. Berthoz, "Biped walking stabilization on soft ground based on gait analysis," in Biomedical Robotics and Biomechatronics (BioRob), 2012 4th IEEE RAS EMBS International Conference on, June 2012, pp. 669-674.

[25] K. Hashimoto, H. j. Kang, M. Nakamura, E. Falotico, H. o. Lim A. Takanishi, C. Laschi, P. Dario. and A. Berthoz, "Realization of biped walking on soft ground with stabilization control based on gait analysis," in Intelligent Robots and Systems (IROS), 2012 IEEE/RSJ International Conference on, Oct 2012, pp. 2064-2069. 